\title{
NEW PROCEDURE/TECHNIQUE
}

\section{Skin Cell Proliferation Stimulated by Microneedles}

\author{
Horst Liebl ${ }^{\mathrm{a}}$, Luther C. Kloth, PT, MS, FAPTA, CWS, FACCWS ${ }^{\mathrm{b}, *}$
}

\author{
${ }^{a}$ Dermaroller ${ }^{\circledR}$ Wolfenbuettel, Germany; and \\ ${ }^{b}$ Department of Physical Therapy, Marquette University, P.O. Box 1881, Milwaukee, WI 53201-1881, USA
}

\section{KEYWORDS: \\ Microneedles; \\ Trans-epithelial \\ potentials; \\ Skin wounds; \\ Hypotrophic scars; \\ Healing phases}

\begin{abstract}
A classical wound may be defined as a disruption of tissue integrity. Wounds, caused by trauma from accidents or surgery, that close via secondary intention rely on the biological phases of healing, i.e., hemostasis, inflammation, proliferation, and remodeling (HIPR). Depending on the wound type and severity, the inflammation phase begins immediately after injury and may last for an average of 7-14 days. Concurrent with the inflammation phase or slightly delayed, cell proliferation is stimulated followed by the activation of the remodeling (maturation) phase. The latter phase can last as long as 1 year or more, and the final healed state is represented by a scar tissue, a cross-linked collagen formation that usually aligns collagen fibers in a single direction. One may assume that skin microneedling that involves the use of dozens or as many as 200 needles that limit penetration to $1.5 \mathrm{~mm}$ over $1 \mathrm{~cm}^{2}$ of skin would cause trauma and bleeding followed by the classical HIPR. However, this is not the case or at least the HIPR phases are significantly curtailed and healing never ends in a scar formation. Conversely dermabrasion used in aesthetic medicine for improving skin quality is based on "ablation" (destruction or wounding of superficial skin layers), which requires several weeks for healing that involves formation of new skin layers. Such procedures provoke an acute inflammatory response. We believe that a less intense inflammatory response occurs following microneedle perforation of the skin. However, the mechanism of action of microneedling appears to be different. Here we review the potential mechanisms by which microneedling of the skin facilitates skin repair without scarring after the treatment of superficial burns, acne, hyperpigmentation, and the non-advancing periwound skin surrounding the chronic ulcerations of the integument.
\end{abstract}

(c) 2013 Elsevier Inc. All rights reserved.

\section{Potential Mechanism of Microneedle Treatment of Normal Skin}

Some explanations can be found with a closer look at enhanced cell communication and motility by endogenous electrical signals (electro-taxis). Dunkin et $\mathrm{al}^{1}$ found that skin cuts to a depth of $0.5-0.6 \mathrm{~mm}$ close by electrical

Conflict of interest: This paper was not adapted from a presentation at a meeting and was not funded by any grant support. The authors report no conflicts of interest.

* Corresponding author.

E-mail address: luther.kloth@marquette.edu cell stimulation without any trace of scar tissue. Zhao et $\mathrm{al}^{2}$ reported similar effects of electrical currents on cell motility and healing. Deeper skin cuts close by "skin repair" that ultimately results in scar formation Figure 1.

In 2010 Liebl proposed that microneedling could be used in treating chronic wounds. In reviewing the literature related to wound healing by electric field stimulation, he theorized that the mechanisms for the main action of microneedling may include trans-epithelial potentials (TEPs) and the skin battery. ${ }^{3}$ Foulds and Barker ${ }^{4}$ placed electrodes on the stratum corneum (SC) and inside the dermis, and measured a negative potential difference of the SC ranging from 10 to $60 \mathrm{mV}$, and averaging $-23.4 \mathrm{mV}$ (Figure 2). 

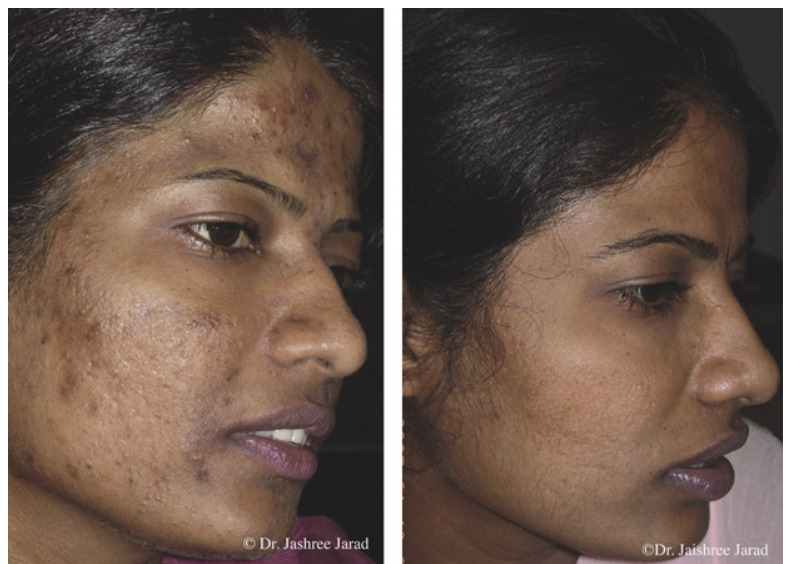

Figure 1 Why and how do skin, scars, acne and hyperpigmentation respond so positively to microneedles? (Three treatments of facial acne each separated by three months, treated with $1.5 \mathrm{~mm}$ needles). Permission to publish granted by Jaishree Sharad.

When a medical grade, non-traumatic microneedle, preferably made from stainless steel, enters the SC and is pushed into the electrolyte of the intercellular space, the only possible reaction is a short circuit of the endogenous electric fields (Figure 3). It must be noted that the needle penetration lasts only fractions of seconds while the microneedles of the device (e.g. Dermaroller ${ }^{\circledR}$ ) roll over the skin. Non-traumatic microneedles with a preferable tip radius of not more than 2$3 \mu \mathrm{m}$ do not create a classical wound that bleeds.

Figuratively speaking, an ordinary hypodermic needle merely "pushes" cells aside. In a classical wound usually bleeding occurs from punctured or cut vessels. In contrast during microneedling there is minimal to no bleeding since only capillaries are punctured. Never-the-less, the mild trauma to the skin results in a mild inflammatory response, likely due to bradykinins and histamine release from mast cells.

After soft tissue injury the $\mathrm{Na} / \mathrm{K}$-pump is activated to reestablish the intra- and extra-cellular electrical potential. ATPase, a trans-membrane protein, delivers positively charged $\mathrm{Na}+$ ions into the intercellular electrolyte and collects $\mathrm{K}+$ ions and transports them into the cell. Charging and discharging of cells takes place in 2-3 milliseconds

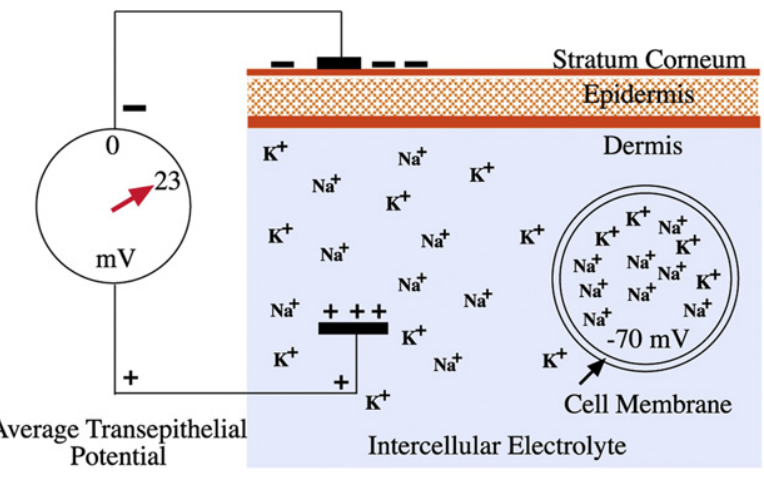

Figure 2 Resting potential.

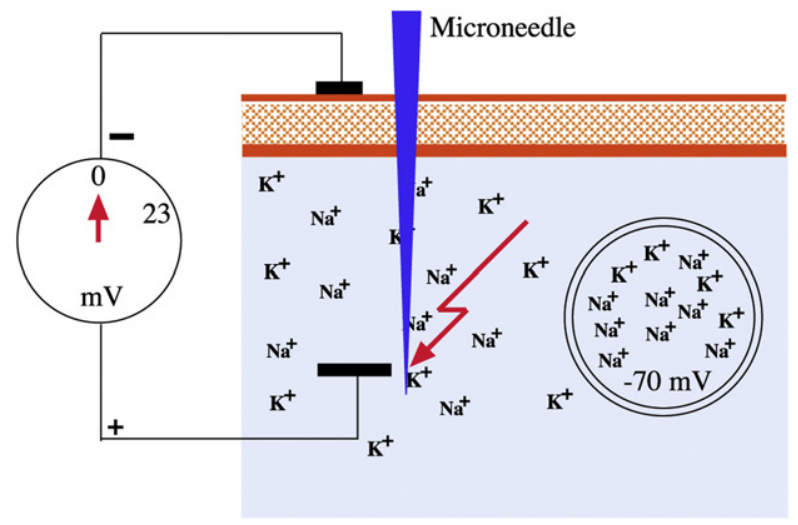

Figure 3 Short circuit.

(Figure 4). However, it must be noted that only cells in the vicinity of the injury (about $2-3 \mathrm{~mm}$ ) are activated. Once the TEP is restored the activated cells resume their resting potential.

To elicit a desired response from microneedling about two hundred needle pricks are created per $\mathrm{cm}^{2}$ of skin. The cells around the needle channels likely sense the reoccurring penetrations as new (repeated) induced wound stimuli and therefore are in a permanent active state that leads to a polarized electro-magnetic field (EMF) in the inter-cellular electrolyte. The EMF stimulates DNA-expression of the surrounding cells. This epigenetic DNA-information by electro-taxis leads to an enhanced motility of epithelial and endothelial cells in the wounded area and subsequently to gene expression of growth factors that facilitate healing (Figure 5).

\section{Potential Mechanism of Microneedle Treatment of Scar Tissue}

Although this article explains what happens after wounding by microneedles, the following information is provided for more detailed understanding. Matrix-MetalloProteinases (MMPs) are thought to play a vital role in cell

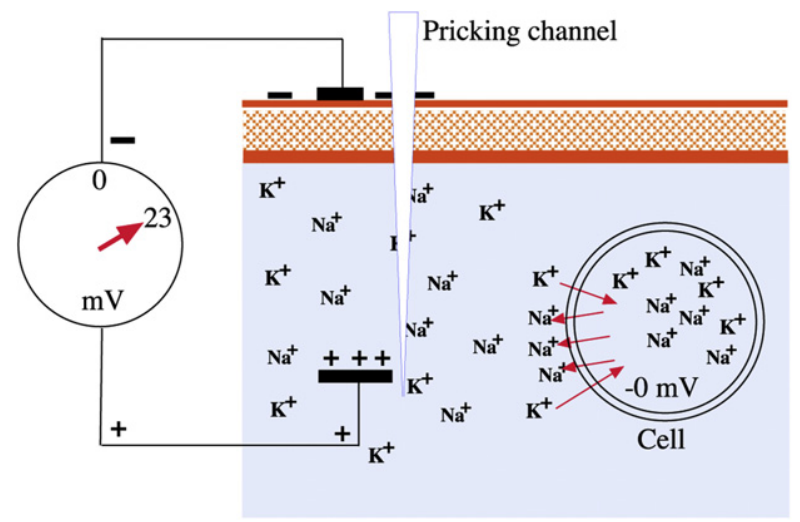

Figure $4 \mathrm{Na}-\mathrm{K}$ pump (Na-K-ATPase starts: $3 \mathrm{Na}$ ions leave; $2 \mathrm{~K}$ ions enter cell). 


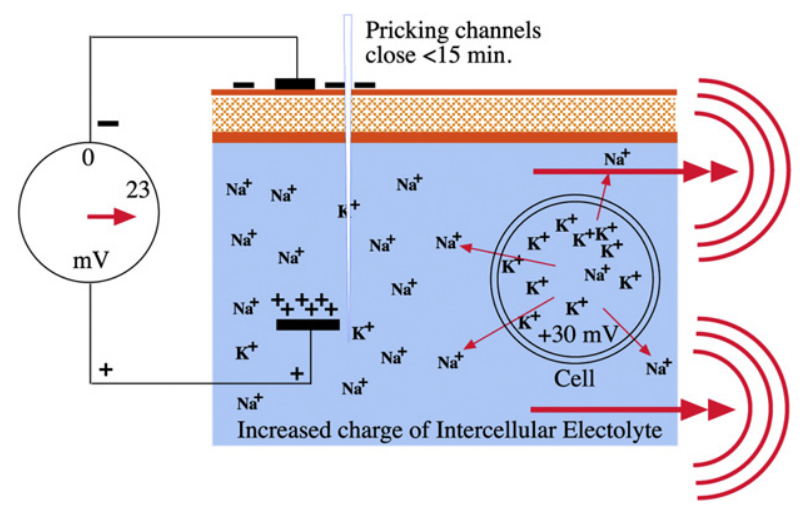

Figure 5 Increased action potential results in an electrical signal.

proliferation, but it is not known if these enzymes are expressed, when normal, non-fibrotic skin is needled. However, we speculate that they play a vital role following scar needling. The formation of scar tissue is controlled by TGF- $\beta 1$ and $\beta 2$. However, Aust et $\mathrm{al}^{5}$ have indicated that after microneedling only TGF- $\beta 3$ seems to control collagen fiber integration into the skin's matrix (Figure 6). MMPs are controlled by inhibitors (TIMPS). They continue to be active to degrade excessive fibrotic tissue until degradation of surplus tissue is complete (Figure 7).

Capillaries and fibroblasts migrate into "former" scar tissue. Synthesized collagen fibers (type III) integrate into the skin matrix. Following microneedling hypotrophic scars "raise" to skin level and former hypertrophic scars "fall" to skin level (Figures 8 and 9). While the new tissue of previously hypotrophic scars requires about 10-20 weeks after 1-3 microneedling treatments with Dermaroller ${ }^{\circledR}$, this process takes several months in hypertrophic scars and burn scars. These scars do not respond to microneedling as well as hypotrophic scars. The failure rate may be around $30 \%$. Research is needed to determine why this difference exists.

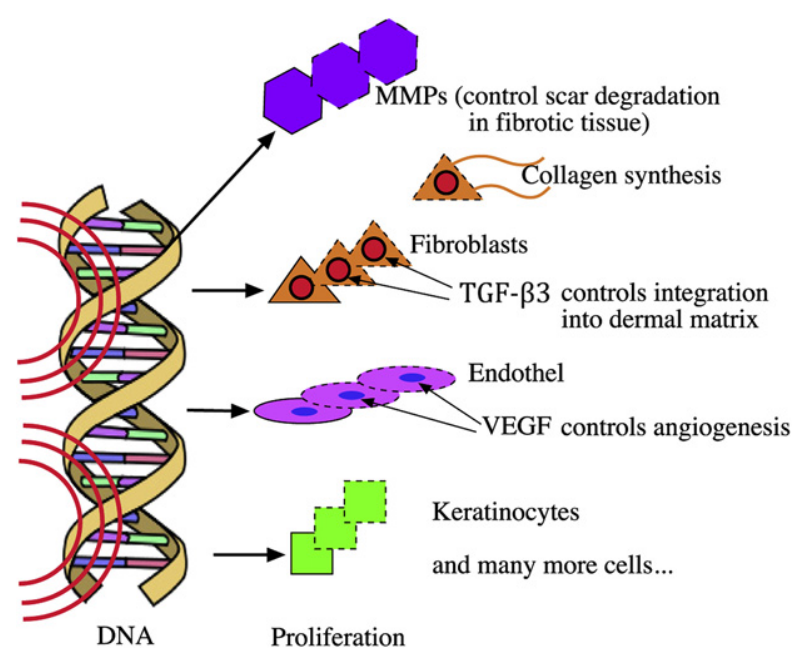

Figure 6 Gene expression - proliferation - synthesis.

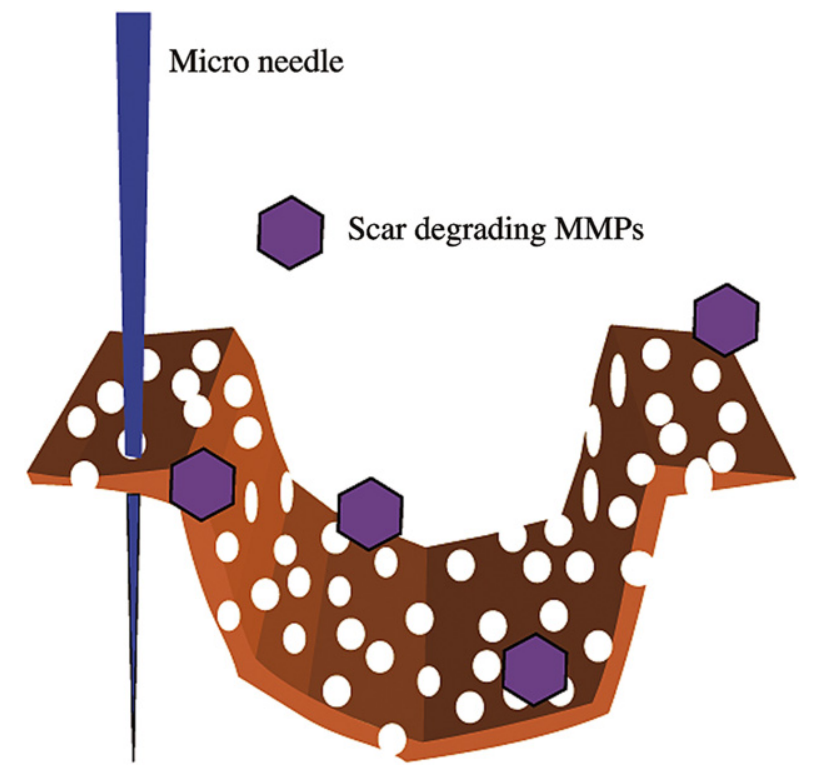

Figure 7 Perforated scar, invading MMPs.

Safonov ${ }^{6}$ reported that keloids respond to microneedling. He treated inactive keloids, but pointed out that a certain minimal risk must be considered. In all of his burn scar cases he emphasized that a long transformation period of up to 8 months may be required depending on the case.

Research results of other methods used to treat cutaneous scars and to create skin sites for autologous cell transplantation.

Interestingly, recent research from the Cochran Database evaluated the effectiveness of silicon gel sheeting for prevention of hypertrophic and keloid scarring in people with newly healed post-surgical wounds and on the effectiveness of established scarring in people with existing keloid or hypertrophic scars. ${ }^{7}$ Thirteen trials involving 559 people, aged from 2 to 81 years were included in the review. The trials compared adhesive silicon gel sheeting with control; non-silicone gel sheeting; silicone gel plates with added vitamin $\mathrm{E}$; laser therapy; triamcinolone acetonide injection and non-adhesive silicone gel sheeting. In the prevention studies when compared with a no treatment

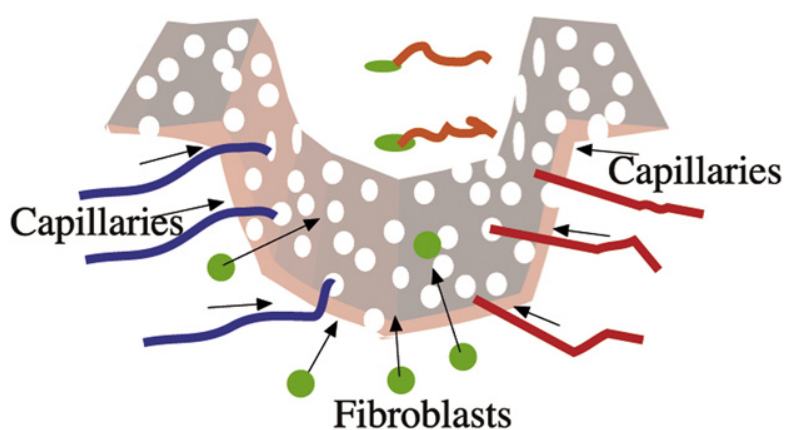

Figure 8 Migrating capillaries and fibroblasts in degrading scar tissue. 
MICRONEEDLE stimulates ANGIOGENESIS by electrical signals and growth factors.

1.

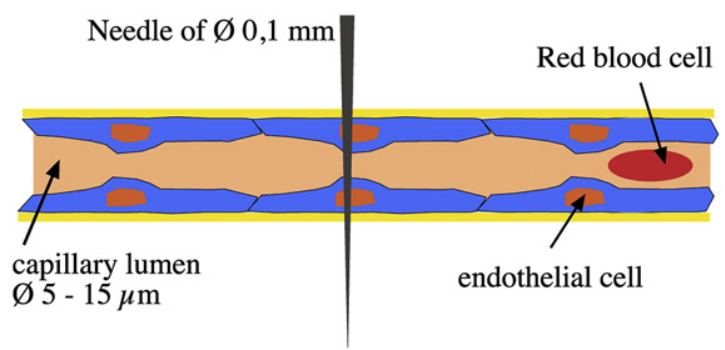

The key molecule of angiogenesis is VEGF (vascular endothelial growth factor)

2.

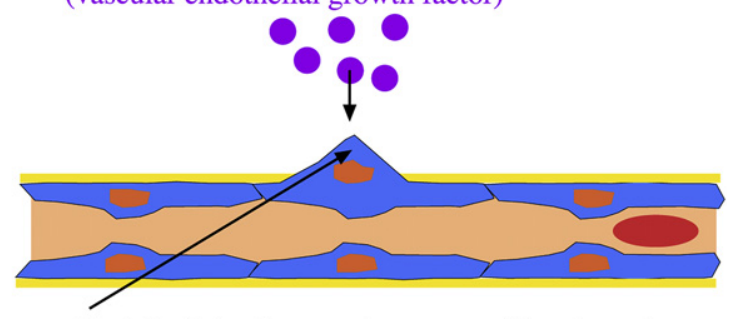

Endothelial cell generates new capillary branch guided by pseudopodial process and stimulated growth factor signals

3.

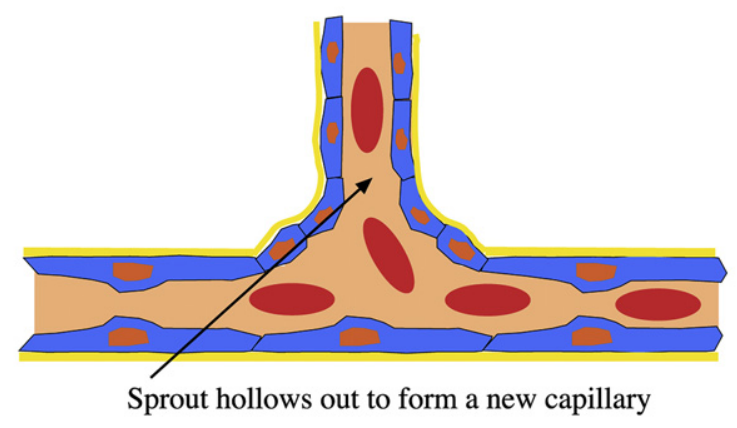

Figure 9 Sprout hollows out to form a new capillary.

option; silicone gel sheeting reduced the incidence of hypertrophic scarring in people prone to scarring (RR 0.46, 95\% CI $0.21-0.98$ ) but these studies were highly susceptible to bias. On the effectiveness of established scarring in people with existing keloid or hypertrophic scars, silicon gel sheeting produced a statistically significant improvement in scar elasticity, (RR 8.60, 95\% CI 2.55-29.02) but these studies were also highly susceptible to bias. Thus, the poor quality research means a great deal of uncertainty prevails regarding the effectiveness of silicon gel sheeting in the prevention and treatment of hypertrophic and keloid scars.

A more noteworthy outcome is reported from a study that compared the characteristics of microscopic treatment zones induced by ablative fractional $\mathrm{CO}_{2}$ laser and by microneedle treatment in ex vivo human breast skin. ${ }^{8}$ While both methods induced minimally invasive sites needed for autologous cell therapy, the $\mathrm{CO}_{2}$ laser resulted in superficial, epidermal papillary dermis defects of $0.1-0.3 \mathrm{~mm}$ covered by a thin eschar coated with denatured collagen. In contrast, the microneedle intervention produced thin vertical skin fissures reaching up to $0.5 \mathrm{~mm}$ into the middermis and injuring dermal blood vessels but without surrounding tissue necrosis. Both technologies created small epidermal defects which allow delivery of isolated cells such as melanocyte transplantation for vitilago, with microneedle treatment having the advantage of lacking devitalized tissue and enabling vascular access for transplanted cells.

\section{Healing Phases After Microneedling}

\section{Inflammation}

The visible inflammation phase (erythema) lasts on average about 48 hours. The redness on Caucasian skin decreases by $50 \%$ 4-6 hours after the treatment. Chilled silk layers (Cool Mask) soaked in hyaluronic acid are extremely helpful in reducing erythema by at least $50 \%$ in 30 minutes. Visible edema is unusual after microneedling. There may be a general slight swelling that fades within 48 hours. In chronic wounds progression toward healing often stalls in the inflammatory phase. At the wound edge, when re-epithelialization is arrested, microneedling of periwound skin may serve to induce a mild inflammatory response which may stimulate epithelial migration to occur. In addition, as previously mentioned the microneedling may help to restore the TEPs and electrotaxis of epithelial skin cells.

\section{Proliferation}

The proliferation phase starts immediately after microneedling and may reach its peak after 2 months. At present it is not known how epidermal and dermal stem cells are affected by microneedling.

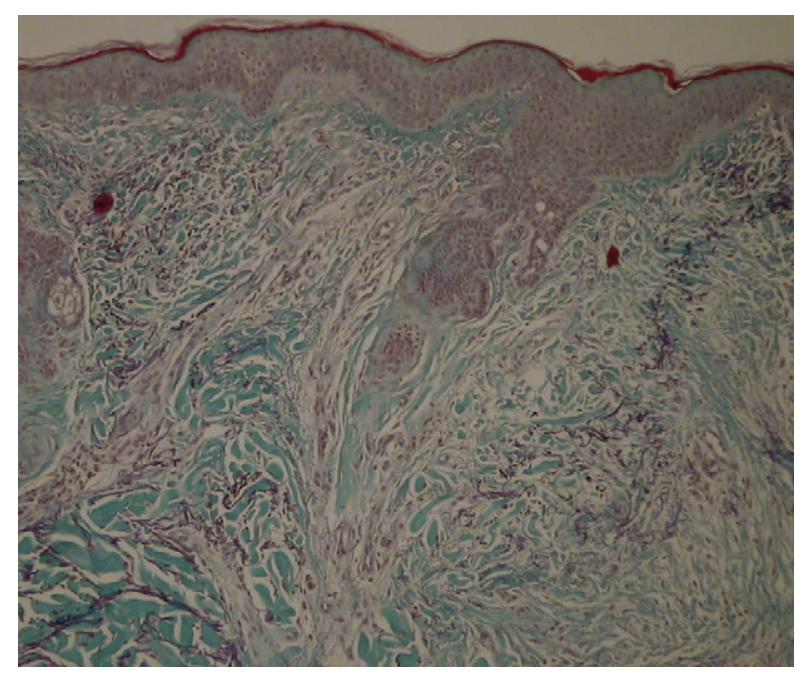

Figure 10 Control biopsy. 


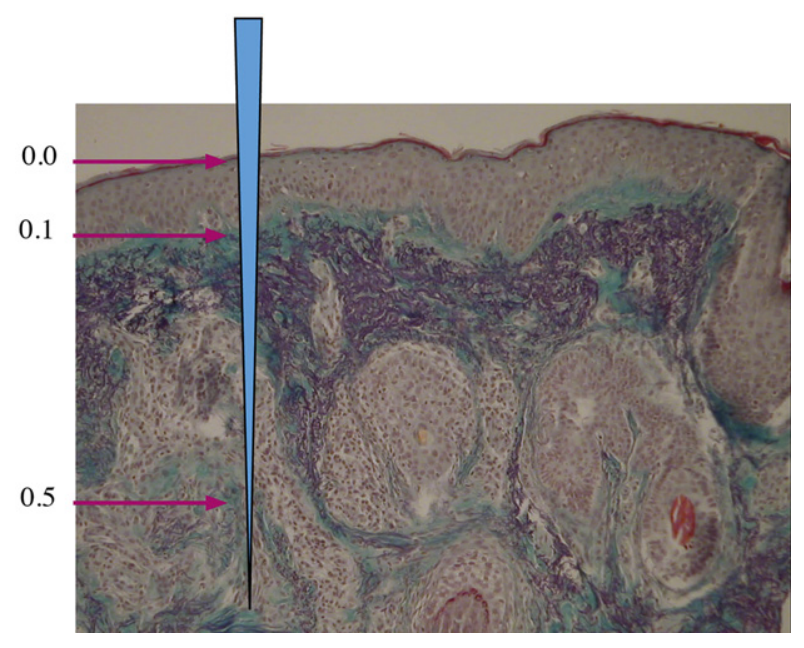

Figure 11 Biopsy from needled skin 6 weeks post-op; collagen stained purple with van Giesen.

\section{Remodeling (maturation)}

New type III collagen fibers integrate into the existing skin matrix without any trace of fibrotic tissue (Compare Figures 10 and 11). An interesting fact is that the new collagen formation is deposited from a depth of $0.6 \mathrm{~mm}$ upwards and towards the basal membrane, in most cases when needles with a length of $1.5 \mathrm{~mm}$ are used. ${ }^{8}$

Skin improvement is evident 3-4 weeks after a microneedle session. ${ }^{9}$ However, collagen maturation needs time, especially to transform into the more elastic collagen type I.

Former atrophic scars show a relatively early improvement that is evident around 2-3 weeks post-needling. As mentioned earlier, the degradation of hypertrophic scars, especially burn scars, may need many months for a visible improvement. Permanent or lasting erythema after thermal exposure responds very well to microneedling. It is assumed that the contraction capabilities of the burned vessel proteins are damaged by heat exposure. MMPs degrade the perforated endothelial cells and stimulate angiogenesis for new capillaries.

We would like to emphasize that in contrast to ablative procedures, post-op infections after microneedling are very unlikely due to the rapid closure of the SC within a maximum of 15 minutes. Bal et al ${ }^{10}$ have not reported any negative side effects in their reports.

\section{Summary}

Microneedling is a fascinating and intriguing new procedure for skin improvement based on induced cell proliferation by electrical signals. We speculate that reduction of hyperpigmentation may be influenced by expression of MMPs, however, research is needed to verify the mechanism(s) involved.

Very good results have been obtained after microneedling of flourishing acne. Acne is triggered by androgens that stimulate increased proliferation of keratinocytes that block the ducts of sebaceous glands. After one or two treatments the hyper proliferation of keratinocytes may be down-regulated. Thus it can only be speculated that MMPs, induced by microneedles, somehow balance or equilibrate cell proliferation.

\section{References}

1. Dunkin CS, Pleat JM, Gillespie PH, Tyler MP, Roberts AH, McGrouther DA: Scarring occurs at a critical depth of skin injury: precise measurement in a graduated dermal scratch in human volunteers. Plast Reconstr Surg. 2007;119(6):1722-1732.

2. Zhao M, Song B, Rajnicek AM: Electrical signals control wound healing through phosphatidylinositol-3-OH kinase-g and PTEN. Nature. 2006;442:457-460.

3. Kloth LC: Electrical stimulation for wound healing: a review of evidence from in vitro studies, animal experiments, and clinical trials. Int J Low Extrem Wounds. 2005;4(1):23-44.

4. Foulds L, Barker A: Human skin battery potentials and their possible role in wound healing. Br J Dermatol. 1983;109:515-522.

5. Aust MC, Reimers K, Vogt PM: Medical needling: improving the appearance of hypertrophic burn-scars. GMS Verbrennungsmedizin. 2009;3:Doc 03 [Please see:http://www.egms.de/static/en/journals/ vmed/2009-3/vmed000007.shtml].

6. Safonov I: Percutaneous collagen induction in correction of post-burn scars. FACE Mag. 2011;1:34-37. [German Edition].

7. O'Brien L, Pandit A: Silicone gel sheeting for preventing and treating hypertrophic and keloid scars. Cochrane Database Syst Rev. 2006;(1): CD003826.

8. Beltraminelli H, Dietrich N, Hunziker T: Fractional transepidermal delivery: a histological analysis. Dermatology. 2011;223(4): 321-324.

9. Schwarz M, Laaff $\mathrm{H}$ : A prospective controlled assessment of micro-needling with the Dermaroller device. Plast Reconstr Surg. 2011;127(6):146e-148e.

10. Bal S, Kruithof AC, Liebl $\mathrm{H}$, et al: In vivo visualization of micro-needle conduits in human skin using laser scanning microscopy. Laser Phys Lett. 2010;7(3):242-246. 